\title{
Bacteriology and Antibiotic Susceptibility Pattern of Urinary Tract Infection in a Tertiary Care Centre
}

\author{
Sarada Dasari*, H. Ravi Chandra Prakash and C. Surya Kumari \\ Department of Microbiology, Viswabharathi medical college, Kurnool, India \\ *Corresponding author
}

\section{Keywords}

Urinary tract infection, Antibiotic susceptibility testing, Antibiotic resistance

Article Info

Accepted:

08 January 2020

Available Online:

10 February 2020

\section{A B S T R A C T}

Urinary tract infection is one of the most common bacterial infections especially in developing countries. It shows lot of regional variation in causative pathogens and antimicrobial susceptibility pattern. Hence requires regular regionwise surveillance. Urine samples received at microbiology laboratory from patients with symptomatology of urinary tract infection were included in the study. All the samples were processed according to standard protocol, identification and antibiotic susceptibility testing was done. Antibiotics tested were ampicillin, cotrimoxazole, amikacin, gentamicin, piperacillintazobactum, ciprofloxacin, imipenem, meropenem, nitrofurantoin, ceftriaxone, cefepime and cefaperazone-sulbactum. Prevalence of urinary tract infection was found to be $33 \%$.female preponderance of $56.08 \%$ was seen over males $43.91 \%$. Patients in the age group of 40-60 years (34.96\%) were found to be more affected. Escherichia coli was the predominant bacteria isolated $(42.22 \%)$.Antibiotic susceptibility profile exhibited least resistance to imipenem, meropenem, nitrofurantoin and amikacin by gram negative bacteria. Gram positive bacteria showed least resistance to vancomycin and linezolid. Treatment to urinary tract infection is usually started empirically. knowledge of susceptibility pattern of common pathogens isolated in this area can guide the physician and help in limiting the antibiotic resistance.

\section{Introduction}

Urinary tract infection is one of the most common bacterial infections that require patients to seek medical attention ${ }^{1}$. Urinary tract infection is bacteriuria with urinary symptoms ${ }^{2}$. It is significant both as community acquired as well as nosocomial infection. Urinary tract infection can be a source of bacteremia. Urinary tract infections involve increased morbidity and associated financial loss. At present it is one of the most common medical conditions encountered in medical practice with the prevalence rate varying from $21.8 \%$ to $31.3 \%$ in various parts of India ${ }^{3}$. Members of enterobacteriaceae are the common causative agents but there are variations in antimicrobial susceptibility and therefore selection of treatment with appropriate antibiotics requires proper laboratory evaluation ${ }^{4}$.

Treatment of urinary tract infection is often started empirically but a large proportion of 
uncontrolled antibiotic usage has contributed to the emergence of resistant bacterial infections ${ }^{5}$. Awareness of local etiology and susceptibility profile could support the most effective empirical treatment ${ }^{6}$.

\section{Materials and Methods}

Present study was done from January 2019 to December 2019 over a period of one year at Viswa Bharathi Medical College, Kurnool.

All the urine samples both from OP and IP patients with symptomatology of UTI received at microbiology laboratory during that period were included in the study. Clean catch midstream urine was collected in a sterile container. Samples were processed by microscopy for pus cells and culture according to standard protocol within 2 hours of collection ${ }^{7}$.

\section{Culture}

Surface streaking on blood agar and MacConkey agar was done by using calibrated loop for semi quantitative method and incubated overnight at $37^{\circ} \mathrm{C}$. After overnight incubation culture plates were examined for growth characteristics and colony count was done. A specimen was considered positive for urinary tract infection if a single organism was cultured at a concentration of $\geq 10^{5} \mathrm{cfu} / \mathrm{ml}$. Lesser counts were also considered significant in symptomatic patients. Identification of the pathogen was done by biochemical reactions like indole production, citrate utilisation test, oxidase test, $\mathrm{H}_{2} \mathrm{~S}$ production, urease test, catalase test, coagulase test, triple sugar iron agar inoculation.

\section{Antibiotic susceptibility testing}

It was done according to CLSI guidelines ${ }^{8}$. Antibiotic discs procured from $\mathrm{Hi}$ media,
India were used. Gentamicin $10 \mu \mathrm{g}$, ampicillin $10 \mu \mathrm{g}$, cotrimoxazole $(1.25 / 23.75$ $\mu \mathrm{g}), \quad$ amikacin $\quad 30 \quad \mu \mathrm{g}$, piperacillintazobactum30/6 $\mu \mathrm{g}$, ciprofloxacin $5 \mu \mathrm{g}$, imipenem $10 \mu \mathrm{g}$, meropenem $10 \mu \mathrm{g}$, nitrofurantoin $30 \mu \mathrm{g}, \quad$ ceftriaxone $\quad 30$ $\mu \mathrm{g}$,cefepime $\quad 30 \quad \mu \mathrm{g}, \quad$ cefaperazonesulbactum $(75 / 30 \mu \mathrm{g})$ were the antibiotic discs used in this study.

Antibiotic susceptibility testing was done on Mueller Hinton agar. Kirby Bauer disc diffusion method was followed. 6-7 antibiotic discs were placed on inoculated MuellerHinton agar plate and incubated at $37^{\circ} \mathrm{C}$ for 18-24 hours.

\section{Statistical analysis}

Calculations were done by using Microsoft excel. Results presented as frequencies and percentages.

\section{Results and Discussion}

The present study was conducted from January 2019 to December 2019 in a tertiary care hospital. A total of 1800 urine samples were processed over a period of one year, out which 592 samples showed significant bacterial growth. The prevalence rate was $33 \%$.

Of the 592 samples which showed significant bacterial growth 332 samples belonged to females and 260 samples were from males. Percentage of females with urinary tract infection was more $(56.08 \%)$ when compared with males $(43.91 \%)($ Table -1$)$

Age wise distribution of urinary tract infection in our study showed higher incidence in the 40-60 years age group (34.96\%) followed by $20-40$ years $(30.23 \%)$ (Table-2). 
Out of the various isolates obtained from the 592 positive cultures Escherichia coli was the commonest $(42.22 \%)$ followed by Klebsiella species $(29.05 \%)$, Pseudomonas aeruginosa (16.55\%), Proteus (6.41\%), Staphylococcus aureus (3.04\%), coagulase negative staphylococci (1.35\%), Enterococcus species (1.01\%), and Citrobacter (0.33\%). (Table-3)

Antibiotic sensitivity profile of the isolated bacteria showed that most of the Gram negative bacteria exhibited least resistance to imipenem, meropenem, nitrofurantoin and amikacin. Among the Gram positive bacteria least resistance was shown by linezolid and vancomycin.

Commonest isolate in our study, Escherichia coli exhibited least resistance tomeropenem (10.4\%), imipenem (12.8\%) followed by nitrofurantoin (22.4\%), amikacin (42.4\%). Maximum resistance was shown by ampicillin.

Staphylococcus aureus which was the commonest Gram positive bacteria isolated exhibited highest sensitivity to vancomycin $(100 \%)$ and linezolid $(100 \%)$. (Table - 4)

Table.1 Gender wise distribution of urinary tract infection

\begin{tabular}{|c|c|c|}
\hline sex & Frequency & Percentage \\
\hline Male & 260 & $43.91 \%$ \\
\hline Female & 332 & $56.08 \%$ \\
\hline
\end{tabular}

Table.2 Age wise distribution of urinary tract infection

\begin{tabular}{|c|c|c|}
\hline Age in years & Frequency & Percentage \\
\hline $\mathbf{0 - 2 0}$ & 45 & $7.6 \%$ \\
$\mathbf{2 0 - 4 0}$ & 179 & $30.23 \%$ \\
$\mathbf{4 0 - 6 0}$ & 207 & $34.96 \%$ \\
$\mathbf{6 0}$ & 161 & $27.19 \%$ \\
\hline
\end{tabular}

Table.3 Distribution of uro pathogens in culture

\begin{tabular}{|c|c|c|}
\hline Organism & Isolation rate & Percentage \\
\hline Escherichia coli & 250 & $42.22 \%$ \\
\hline Klebsiella species & 172 & $29.05 \%$ \\
\hline Pseudomonas aeruginosa & 98 & $16.55 \%$ \\
\hline Proteus species & 38 & $6.41 \%$ \\
\hline Citrobacter species & 2 & $0.33 \%$ \\
\hline Staphylococcus aureus & 18 & $3.04 \%$ \\
\hline Coagulase negative staphylococci & 8 & $1.35 \%$ \\
\hline Enterococci & 6 & $1.01 \%$ \\
\hline Total & $\mathbf{5 9 2}$ & \\
\hline
\end{tabular}


Table.4 Resistance pattern of antibiotics against uropathogens

\begin{tabular}{|c|c|c|c|c|c|c|c|c|c|c|c|c|}
\hline Organism & Amp & COT & $\mathbf{A k}$ & Gen & PIT & Cip & IPM & Mrp & NIT & CTR & CPM & CFS \\
\hline $\begin{array}{l}\text { Escherichia } \\
\text { coli } \\
(250)\end{array}$ & $\begin{array}{c}223 \\
(89.2 \%)\end{array}$ & $\begin{array}{c}186 \\
(74.4 \%)\end{array}$ & $\begin{array}{c}106 \\
(42.4 \%)\end{array}$ & $\begin{array}{c}112 \\
(44.8 \%)\end{array}$ & $\begin{array}{c}122 \\
(48.8 \%)\end{array}$ & $\begin{array}{c}151 \\
(60.4 \%)\end{array}$ & $\begin{array}{c}32 \\
(12.8 \%)\end{array}$ & $\begin{array}{c}26 \\
(10.4 \%)\end{array}$ & $\begin{array}{c}56 \\
(22.4 \%)\end{array}$ & $\begin{array}{c}156 \\
(62.4 \%)\end{array}$ & $\begin{array}{c}192 \\
(76.8 \%)\end{array}$ & $\begin{array}{c}146 \\
(58.4 \%)\end{array}$ \\
\hline $\begin{array}{l}\text { Klebsiella } \\
\text { (172) }\end{array}$ & $\begin{array}{c}156 \\
(90.69 \%)\end{array}$ & $\begin{array}{c}128 \\
(74.41 \%)\end{array}$ & $\begin{array}{c}78 \\
(45.34 \%)\end{array}$ & $\begin{array}{c}82 \\
(47.67 \%)\end{array}$ & $\begin{array}{c}69 \\
(40.11 \%)\end{array}$ & $\begin{array}{c}118 \\
(68.6 \%)\end{array}$ & $\begin{array}{c}34 \\
(19.76 \%)\end{array}$ & $\begin{array}{c}28 \\
(16.27 \%)\end{array}$ & $\begin{array}{c}46 \\
(26.74 \%)\end{array}$ & $\begin{array}{c}106 \\
(61.62 \%)\end{array}$ & $\begin{array}{c}126 \\
(73.25 \%)\end{array}$ & $\begin{array}{c}92 \\
(53.48 \%)\end{array}$ \\
\hline $\begin{array}{l}\text { Pseudomon } \\
\text { as } \\
(98)\end{array}$ & - & - & $\begin{array}{c}49 \\
(50 \%)\end{array}$ & $\begin{array}{c}51 \\
(52 \%)\end{array}$ & $\begin{array}{c}52 \\
(53.06 \%)\end{array}$ & $\begin{array}{c}76 \\
(77 \%)\end{array}$ & $\begin{array}{c}48 \\
(48.9 \%)\end{array}$ & $\begin{array}{c}45 \\
(45.91 \%)\end{array}$ & - & - & $\begin{array}{c}74 \\
(75.51 \%)\end{array}$ & $\begin{array}{c}56 \\
(57.14 \%)\end{array}$ \\
\hline $\begin{array}{l}\text { Proteus } \\
(38)\end{array}$ & $\begin{array}{c}35 \\
(92.1 \%)\end{array}$ & $\begin{array}{c}32 \\
(84.21 \%)\end{array}$ & $\begin{array}{c}21 \\
(55.26 \%)\end{array}$ & $\begin{array}{c}25 \\
(65.7 \%)\end{array}$ & $\begin{array}{c}19 \\
(50 \%)\end{array}$ & $\begin{array}{c}22 \\
(57.8 \%)\end{array}$ & $\begin{array}{c}17 \\
(44.7 \%)\end{array}$ & $\begin{array}{c}16 \\
(42.1 \%)\end{array}$ & - & $\begin{array}{c}29 \\
(76.31 \%)\end{array}$ & $\begin{array}{c}27 \\
(71.05 \%)\end{array}$ & $\begin{array}{c}26 \\
(68.42 \%)\end{array}$ \\
\hline \multirow[t]{2}{*}{$\begin{array}{l}\text { Citrobacter } \\
\text { (2) }\end{array}$} & $\begin{array}{c}2 \\
(100 \%)\end{array}$ & $\begin{array}{c}2 \\
(100 \%)\end{array}$ & $\begin{array}{c}1 \\
(50 \%)\end{array}$ & $\begin{array}{c}1 \\
(50 \%)\end{array}$ & 0 & $\begin{array}{c}1 \\
(50 \%)\end{array}$ & 0 & 0 & $\begin{array}{c}1 \\
(50 \%)\end{array}$ & $\begin{array}{c}1 \\
(50 \%)\end{array}$ & $\begin{array}{c}1 \\
(50 \%)\end{array}$ & $\begin{array}{c}1 \\
(50 \%)\end{array}$ \\
\hline & Amp & COT & $\mathbf{V a}$ & $\mathbf{L z}$ & Cip & $\mathbf{L E}$ & AK & & & & & \\
\hline $\begin{array}{l}\text { Staphylococ } \\
\text { cus aureus } \\
(\mathbf{1 8 )}\end{array}$ & $\begin{array}{c}13 \\
(72.22 \%)\end{array}$ & $\begin{array}{c}11 \\
(61.11 \%)\end{array}$ & 0 & 0 & $\begin{array}{c}8 \\
(44 \%)\end{array}$ & $\begin{array}{c}10 \\
(55.5 \%)\end{array}$ & $\begin{array}{c}11 \\
(61.11 \%)\end{array}$ & & & & & \\
\hline $\begin{array}{l}\text { CNS } \\
(8)\end{array}$ & $\begin{array}{c}6 \\
(75 \%)\end{array}$ & $\begin{array}{c}5 \\
(62.5 \%)\end{array}$ & 0 & 0 & $\begin{array}{c}4 \\
(50 \%)\end{array}$ & $\begin{array}{c}3 \\
(37.5 \%)\end{array}$ & $\begin{array}{c}5 \\
(62.5 \%)\end{array}$ & & & & & \\
\hline $\begin{array}{l}\text { Enterococci } \\
\text { (6) }\end{array}$ & $\begin{array}{c}5 \\
(83.3 \%)\end{array}$ & $5(83.3 \%)$ & $1(16.6 \%)$ & $\begin{array}{c}2 \\
(33.3 \%)\end{array}$ & $\begin{array}{c}4 \\
(66.6 \%)\end{array}$ & $\begin{array}{c}3 \\
(50 \%)\end{array}$ & $\begin{array}{c}4 \\
(66.6 \%)\end{array}$ & & & & & \\
\hline
\end{tabular}


Urinary tract infection is one of the common causes for which medical attention is sought. Hence this study was taken up to know the prevalence rate and to guide in the selection of antibiotic with higher sensitivity.

Prevalence rate in our study was found to be $33 \%$. Similar prevalence rates were found in other studies by Carolin Elizabeth George ${ }^{3}$ (32.1\%), T.S Sailaja et al ${ }^{9}$ (33.5\%), JharnaMandal $^{10}(26.01 \%)$. Where as in some studies prevalence was either less GetenetBeyene $^{5} 9.2 \%$ or more Das RN ${ }^{11}(71.7 \%)$.

Female preponderance over males which was observed in the present study females $(56.08 \%)$ males $(43.91 \%)$ was also observed in studies by V. Rajendran ${ }^{12}$, (females $68.63 \%$, males $31.36 \%$ ), JubinaBency $\mathrm{A}^{13}$ females $63.3 \%$, males $36.7 \%$ ), Vijay Prakashsingh ${ }^{14}$ (females $45.4 \%$, males $22.3 \%$ ).

In the present study higher incidence of urinary tract infection was observed in the age group of 40-60 years $(34.96 \%)$. Similar observation was made by Das R N ${ }^{11}(31.4 \%)$.

Escherichia coli was the most common bacteria isolated in this study (42.22\%). It was followed by klebsiella species (29.05\%), Pseudomonas aeruginosa (16.55\%) similar pattern was also seen in many studies by Harsh kumar B Patel ${ }^{15}$ where in Escherichia coli $(36.11 \%)$, Klebsiella species (18.06\%) were reported, study by Atit shah ${ }^{16}$ shows Escherichia coli (51.88\%), Klebsiella species $(19.55 \%)$, frequency of Escherichia coli (61.42\%), Klebsiella species (14.22\%), Pseudomonas aeruginosa $(8.02 \%)$ was seen in study by PareveeDalal ${ }^{17}$. Gram negative bacteria isolated in our study exhibited least resistance to imipenem, meropenem, nitrofurantoin and amikacin and highest resistance to ampicillin. Among Gram positive bacteria least resistance was shown by vancomycin and linezolid, highest resistance by ampicillin. similar observations in antibiotic susceptibility pattern were made in other studies by JubinaBency A ${ }^{13}$, Vijay Prakashsingh $^{14}$ and Paravee Dalal ${ }^{17}$.

In conclusion bacterial uropathogens show regional variation in causative agents and their antimicrobial susceptibility pattern. The present study provides the data required to analyze the prevalence rate, most common bacteria implicated in causing urinary tract infection in this region. It also helps to study the pattern in the antimicrobial resistance. Increasing susceptibility to nitrofurantoin can be observed in this study. Due to maximum resistance exhibited by ampicillin it can be avoided in the emipirical treatment for urinary tract infection. Routine surveillance of antibiotic susceptibility can prevent antibiotic misuse and can aid in controlling antibiotic resistance.

\section{Acknowledgement}

We would like to express our gratitude to Viswa Bharathi medical college and hospital, Head of the department of Microbiology, Technical staff of microbiology department.

\section{List of antibiotics used in the study}

Amp-Ampicillin, COT- cotrimoxazole, AKamikacin, Gen-Gentamicin, PIT-Piperacillintazobactum, Cip-Ciprofloxacin, IpmImipenem, Mrp- Meropenem, NITNitrofurantoin, CTR- ceftriaxone, CPMCefepime, CFS- cefaperazone-sulbactum, VaVancomycin, Lz- Linezolid, CipCiprofloxacin, Le- levofloxacin.

\section{References}

1. Diagnostic Microbiology. Bailey and Scotts. $12^{\text {th }}$ ed. Mosby Elsevier publication. 
2. Perpetua A Ekwealor, Malachy C Ugwu et al.2016.Antimicrobial evaluation of bacterial isolates from urine specimen of patients with complainants of urinary tract infection in Awaka, Nigeria.Int $\mathbf{J}$ microbiology.http://dx.doi.org/10.1155/ 2016/9740273.

3. Carolin Elizabeth George, Gift Norman, G. VenkataRamana ,devashriMukharjee and Tata Rao.2015.Treatment of uncomplicated symptomatic urinary tract infections: Resistance patterns and misuse of antibiotics. J Family Med Prim Care.Jul-Sep; 4(3): 416-421

4. Medical microbiology.A guide to microbial infections. David Greenwood, Richard slack, Mike Barer, Will Irving. $18^{\text {th }}$ ed. Elsevier publications.

5. GetenetBeyene, WondewosenTsegaye et al. 2011. Bacterial uropathogens in urinary tract infection and antibiotic susceptibility pattern in Jimmauniversity specialized hospital, southwest Ethiopia.Ethio J Health Sci. Jul; 21(2): 141-146.

6. Farajnia S, Alikhani MY, Ghotaslou R, Naghili B,Nakhlband A, C. 2009. Causative agents and antimicrobial susceptibilities of urinary tract infection in the northwest of Iran.Int $\mathbf{J}$ Infect Dis.13: 140-144.

7. Mackie and McCartney Practical medical microbiology. Collee JG, Fraser AG, et al. $14^{\text {th }}$ ed. Churchill Livingstone New York.

8. Clinical and Laboratory standards Institute, performance standards for antimicrobial susceptibility testing. $26^{\text {th }}$ ed. CLSI supplement M100S. Wayne, PA: Clinical and Laboratory standards Institute; 2016.

9. T. S. Sailaja, A. Mohan Kumar et al. 2017.Bacteriological profile of urinary tract infection in a tertiary care centre. Indian J Microbiol Res. 4(3): 328-332.

10. JharnaMandal, N. Srinivas Acharya.2012.
Antibiotic resistance pattern among common bacterial uropathogens with a special reference to ciprofloxacin resistant Escherichia coli.Indian $\mathrm{J}$ Med Res.136 (11): 842-49.

11. Das RN, Chandrashekar Joshi HS et al.2006.Frequency and susceptibity profile of pathogens causing urinary tract infection at a tertiary care hospital in western Nepal. TS, Singapore Med $\mathrm{J} ; 47(4): 281$.

12. V. Rajendran,R. Napolean, Prashant V Solanke.2017.A study on urinary tract infection in a tertiary care hospital.Int $\mathbf{J}$ Adv Med 2017 oct;4(5): 1401-1405.

13. JubinaBency A.T, Priyanka $R$ et al. 2017.A study on the bacteriological profile of urinary tract infection in adults and their antibiotic sensitivity pattern in a tertiary care hospital in central kerala, India. Int J Res Med Sci 2017 feb;5(2)666-669.

14. Vijay Prakash Singh, Abhishek Mehtaet al.2017. Bacteriological profile of urinary tract infections at a tertiary care hospital in western Uttar Pradesh, India. Int J Res Med Sci. 2017 May;5(5): 2126-2129.

15. Harsh Kumar B Patel, Sumeeta T Soni. Jet al.2019. Causative agents of urinary tract infection and their antimicrobial susceptibility patterns at a referral centre in western India.An audit to help clinicians prevent antibiotic misuse. Family Med Prim Care.8(1):154-159.

16. Atit Shah, MitalVinzuda et al.2015.Clinic-microbiological profile of urinary tract infection in tertiary care hospital in Ahmedabad, Gujarat, India.. Int J current Microbiol. App Sci. 4(9): 288-298.

17. PareveeDalal, JayashriPethaniet al.2016.Microbiological Profile of urinary tract infection in a tertiary care hospital.Journal of research in medical and dental science. Vol 4(3), july-sep. 


\section{How to cite this article:}

Sarada Dasari, H. Ravi Chandra Prakash and Surya Kumari, C. 2020. Bacteriology and Antibiotic Susceptibility Pattern of Urinary Tract Infection in a Tertiary Care Centre. Int.J.Curr.Microbiol.App.Sci. 9(02): 1205-1211. doi: https://doi.org/10.20546/ijcmas.2020.902.142 